\title{
Finanzsystem und wirtschaftliche Entwicklung in den USA und in Deutschland im Vergleich - Eine makroökonomische Skizze
}

\author{
Till van Treeck \\ Eckhard Hein \\ Petra Dünhaupt
}

\begin{abstract}
Die in diesem Jahr durch die Krise am US-amerikanischen Immobilienmarkt ausgelösten Turbulenzen auf den internationalen Finanzmärkten verdeutlichen die realwirtschaftlichen Gefahren eines finanzmarktzentrierten Wachstumsmodells. Ein solches Modell hat sich insbesondere in den USA schon sehr weit entwickelt und scheint dort mittlerweile an Grenzen zu stoßen. Erste Ansätze einer finanzmarktgetriebenen Entwicklung zeigen sich seit einiger Zeit auch in Deutschland, allerdings mit einer deutlich geringeren Wachstumsdynamik. Der Beitrag umreißt die längerfristigen makroökonomischen Triebkräfte in den USA sowie die dort angelegten Instabilitätspotenziale und identifiziert Ansätze und mögliche Probleme einer verstärkten Finanzmarktorientierung in Deutschland. ${ }^{1}$
\end{abstract}

\section{Einführung}

Die Turbulenzen am US-amerikanischen Immobilienmarkt legen die Befürchtung nahe, dass hiermit sowohl für die USA selbst als auch für die Weltwirtschaft insgesamt, und damit auch für Deutschland, negative Folgen für Konjunktur, Wachstum und Beschäftigung verbunden sein könnten. Die Geschehnisse am Immobilienmarkt in den USA sind dabei nur eine Erscheinungsform eines grundlegenden Strukturwandels der US-amerikanischen Volkswirtschaft, welcher bisweilen mit dem Schlagwort „Finanzialisierung“ umschrieben wird.

Mit dem Begriff „Finanzialisierung“ bezeichnen wir allgemein einen Bedeutungszuwachs des finanziellen Sektors einer Volkswirtschaft gegenüber dem nichtfinanziellen Sektor. Dies kommt zunächst in einer Liberalisierung und Deregulierung des Finanzsystems mit dem Ergebnis einer zunehmenden Bedeutung von (immer vielfältigeren) marktfähigen Wertpapieren gegenüber traditionellen Bankkrediten zum Ausdruck. Für den nichtfinanziellen Unternehmenssektor bedeutet ,Finanzialisierung" zum einen, dass das Management von Aktiengesellschaften sein Augenmerk zunehmend auf Finanzmarktkennzahlen legen muss (Shareholder Value-Orientierung). Zugleich werden Industrieunternehmen in verstärktem Maße selbst zu Fi- nanzanlegern: Nachdem sie sich traditionell weitgehend auf die physische Investitionstätigkeit beschränkten, gewinnen nun Aktien(rück)käufe, feindliche Übernahmen und Portfoliomanagement an Bedeutung. Aus Sicht der privaten Haushalte steigt im Zuge der "Finanzialisierung“ die Bedeutung von Finanzvermögen (Wertpapiere, Immobilien) und Kapitaleinkommen gegenüber dem Arbeitseinkommen. Gleichzeitig nehmen durch die Liberalisierung des Kreditgeschäfts die Möglichkeiten der vermögensbasierten Schuldenfinanzierung von Konsum zu. Zudem trägt Shareholder Value-Orientierung potenziell zu größerer Einkommensungleichheit bei.

Der Startpunkt der „Finanzialisierung“ der amerikanischen Volkswirtschaft wird gemeinhin in den frühen 1980er Jahren gesehen. Im Hinblick auf Deutschland bezeichnet „Finanzialisierung“ ein noch sehr junges und im Vergleich zu den USA weniger stark ausgeprägtes Phänomen, welches jedoch spätestens seit der Euphorie des „Neuen Marktes“ Ende der 1990er Jahre und den etwa gleichzeitig einsetzenden institutionellen Deregulierungsmaßnahmen an Bedeutung gewinnt (Windolf 2005; EZB 2007).

In diesem Beitrag sollen die Chancen und Risiken der „Finanzialisierung“ am Beispiel der USA und Deutschlands erörtert werden. Im Unterschied zu der Mehrzahl der bisherigen Studien, die in der Regel unternehmenszentriert bzw. partialanalytisch argumentieren, wählen wir dabei eine explizit makroökonomische und kreislauftheoretische Perspektive.

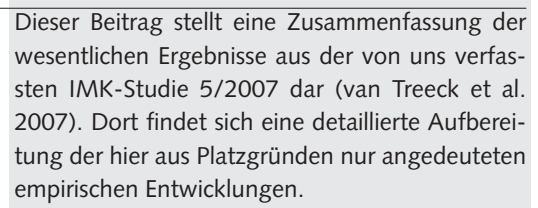
wesentlichen Ergebnisse aus der von uns verfassten IMK-Studie 5/2007 dar (van Treeck et al. 2007). Dort findet sich eine detaillierte Aufbereitung der hier aus Platzgründen nur angedeuteten empirischen Entwicklungen.

Till van Treeck, Doktorand an der Carl von Ossietzky Universität Oldenburg und am Institut für Makroökonomie und Konjunkturforschung (IMK) in der Hans-BöcklerStiftung, Projekt "Finanzsystem und Wirtschaftswachstum".

e-mail: till-van-treeck@boeckler.de Eckhard Hein, Dr., Referatsleiter am Institut für Makroökonomie und Konjunkturforschung (IMK) in der Hans-Böckler-Stiftung, Privatdozent an der Carl von Ossietzky Universität Oldenburg, Gastprofessor an der Wirtschaftsuniversität Wien und an der Universität Hamburg.

Arbeitsschwerpunkte: Geld, Verteilung und Wachstum; Europäische Wirtschaftspolitik; Post-Keynesianische Makroökonomie. e-mail: eckhard-hein@boeckler.de Petra Dünhaupt, Studentin an der Fachhochschule für Technik und Wirtschaft Berlin und Praktikantin am IMK in der Hans-BöcklerStiftung, Projekt "Finanzsystem und Wirtschaftswachstum".

e-mail: petra-duenhaupt@boeckler.de 


\section{"Finanzialisierung ${ }^{\prime \prime}$, reale Investitionen und Profite}

In der akademischen Literatur (und in der politischen Debatte) zu den mikro- und makroökonomischen Effekten von „Finanzialisierung" im Sinne unserer Eingrenzung stehen sich im Wesentlichen zwei Positionen gegenüber:

Nach einer ersten Sichtweise, die sich theoretisch aus einer Integration von Erkenntnissen der neuen Wachstumstheorie und der Informationsökonomie ergibt, wirkt sich der Entwicklungsgrad des Finanzsystems und insbesondere der Finanzmärkte tendenziell positiv auf Spar- und Investitionstätigkeit und damit auf Wirtschaftswachstum und Beschäftigung aus (Hein 2005; Levine 2005). Hierbei wird, wie in der neoklassischen Wachstumstheorie üblich, die Höhe der Vollbeschäftigungsersparnis den Investitionen vorausgesetzt. ${ }^{2}$ Hochentwickelte Finanzmärkte sollen durch attraktive Anlagemöglichkeiten zum einen die private Ersparnis und damit das Kapitalangebot für reale Investitionen vergrößern. Zum anderen sollen „effiziente“ Finanzmärkte die Ersparnisverwendung durch produktive Investitionen verbessern. Insbesondere die laufende Unternehmensbewertung über den Aktienmarkt führt demnach dazu, dass das Unternehmensmanagement dauerhaft zu einer effizienten Verwendung von Finanzierungsmitteln diszipliniert wird, zumal wenn die Bezahlung der Manager an den Aktienwert gekoppelt ist (Jensen/ Meckling 1976).

In einer zweiten, alternativen Sichtweise wird potenziellen makroökonomischen Risiken der Finanzmarktorientierung größere Beachtung geschenkt. Hierbei stehen die unternehmerischen Investitionen als wichtiger Teil der effektiven Nachfrage und Grundlage für das Wachstum der Produktionskapazitäten im Mittelpunkt. ${ }^{3}$ Eine ausgeprägte Shareholder Value-Orientierung kann sich demnach negativ auf die Investitionsneigung von Unternehmen und damit auf Wachstum und Beschäftigung auswirken. Zum einen geht Shareholder Value-Orientierung häufig mit einer verstärkten Gewinnausschüttung an die Aktionäre einher (Dividenden, Aktienrückkäufe). Dies wirkt ceteris paribus investitionshemmend, da angesichts unvoll-

Abb. 1: Investitionen, Profite und Aktienkurse, USA und Deutschland seit $1960(1980=100)$

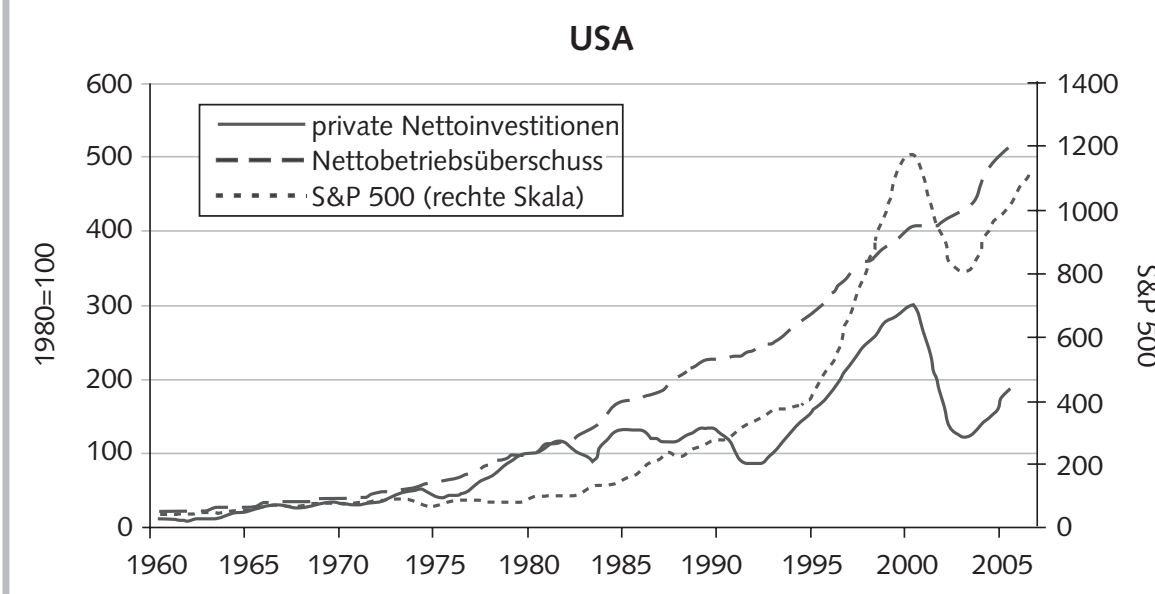

\section{Deutschland}

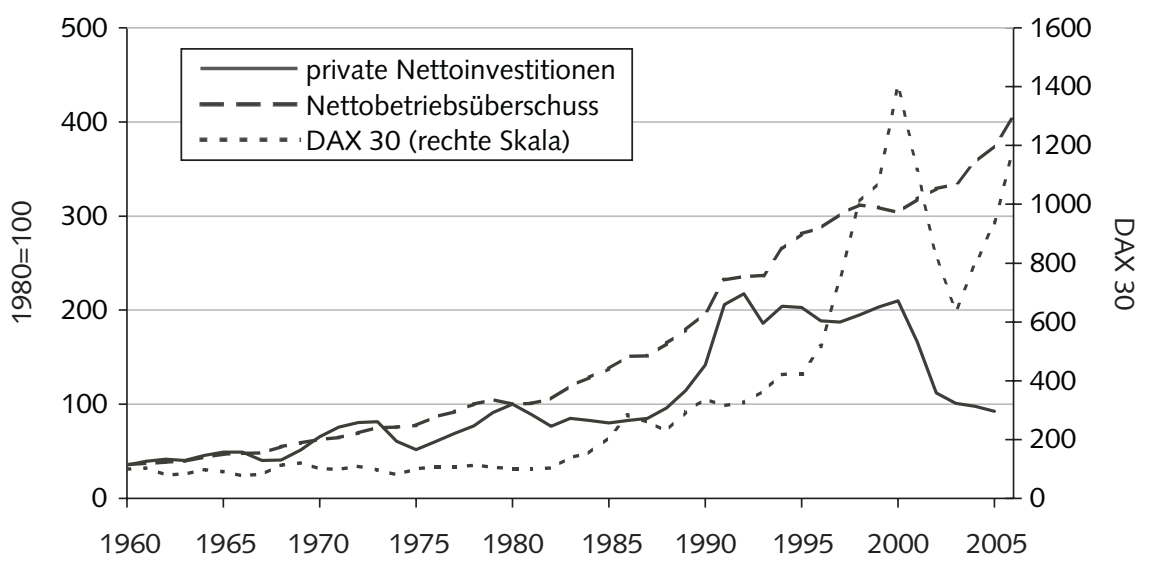

Anmerkungen: USA: private Nettoinvestitionen ohne Wohnungsbau; Nettobetriebsüberschuss, private Unternehmen; S\&P 500, Jahresendstände. Deutschland: Nettofixkapitalbildung, privater Sektor; Nettobetriebsüberschuss, gesamte Volkswirtschaft; DAX 30, jahresdurchschnittliche Monatsendstände. Quelle: NIPA, AMECO, Ecowin; Berechnungen und Darstellung der Autoren.

WSI MitTEILUNGEN

ständiger Finanzmärkte eine starke positive Korrelation zwischen internen Finanzierungsmitteln und Investitionsausgaben der Unternehmen feststellbar ist. ${ }^{4}$ Überdies führen Informationsbeschaffungskosten dazu, dass Aktionäre sich bei ihren Anlageentscheidungen in der Regel an kurzfristigen Unternehmensergebnissen orientieren, sodass Manager in hohem Maße selbst längerfristig wertsteigernde Investitionen unterlassen, wenn sich hierdurch z. B. das „Ergebnis pro Aktie“ kurzzeitig verschlechtert (z. B. Graham et al. 2005).

Tatsächlich zeigt sich seit den 1980er Jahren in den USA und in Deutschland eine tendenzielle Abschwächung der gesamtwirtschaftlichen Investitionsdynamik insbesondere im Verhältnis zur Entwicklung der gesamtwirtschaftlichen Gewinne (Abbildung 1). Für beide Länder lässt sich dabei für die vergangenen Jahrzehnte eine grobe Einteilung in zwei Perioden vornehmen:

(1) Im Zeitraum zwischen den frühen 1960er Jahren und den frühen 1980er Jahren haben sich Investitionen und Gewinne in beiden Ländern weitgehend parallel entwickelt und sind relativ kontinuierlich gewachsen. Gleichzeitig war die Aktienkurs-

2 Vgl. den Überblick in Hein (2004, S. 117 ff.).

3 Die gesamtwirtschaftliche Ersparnis ergibt sich hierbei erst im Nachhinein durch Einkommensund Verteilungseffekte. Zu einem Überblick über die post-keynesianische Verteilungs- und Wachstumstheorie vgl. Hein (2004, S. 133ff.).

4 Vgl. zum Zusammenhang von internen Finanzierungsmitteln und Investitionen z. B. Corbett/Jenkinson (1997); Hubbard (1998); Akerlof (2007). 


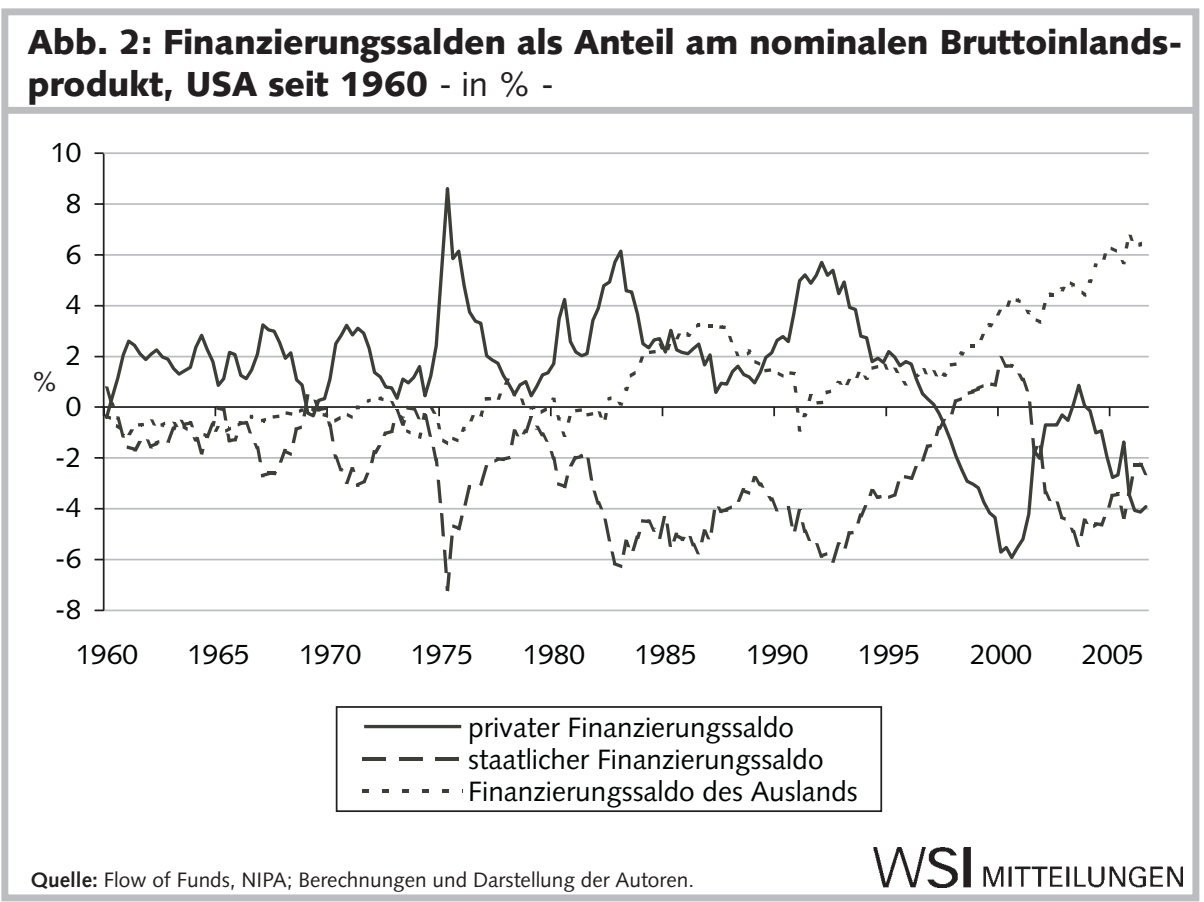

dynamik in beiden Ländern über den gesamten Zeitraum schwach.

(2) Seit den frühen 1980er Jahren hat sich der Wert der Aktienindizes in beiden Ländern mehr als verzehnfacht, während sich das Investitionswachstum über weite Strecken verlangsamt hat und vor allem deutlich hinter dem Anstieg der Unternehmensgewinne zurückgeblieben ist. In den USA wurde diese Auseinanderentwicklung durch den „New Economy“-Boom Ende der 1990er Jahre unterbrochen, in Deutschland durch den Vereinigungs-Boom Anfang der 1990er Jahre.

Auf Grundlage dieser groben Periodisierung scheint also wenig dafür zu sprechen, dass die Finanzmarktliberalisierung seit Anfang der 1980er Jahre die realwirtschaftliche Investitionstätigkeit dauerhaft positiv beeinflusst hätte. Zudem stellt die aus Abbildung 1 ersichtliche zeitweise „Entkoppelung“" von realer Investitionstätigkeit und Unternehmensgewinnen ein erklärungsbedürftiges makroökonomisches Phänomen dar. Aus Sicht eines einzelnen Unternehmens mag zwar, wie oben angedeutet, in der Tat bisweilen ein Zielkonflikt zwischen Gewinnen und Investitionen bestehen, wobei Shareholder Value-Orientierung zu einer höheren Gewichtung des Profitziels führen dürfte. Auf makroökonomischer Ebene gilt dieser $\mathrm{Zu}$ sammenhang jedoch so nicht, da hier die Investitionsausgaben nicht nur Kosten für die Unternehmen, sondern auch einen wichtigen Teil der Güternachfrage darstellen. Es gilt dabei folgende Bestimmungsgleichung für die gesamtwirtschaftlichen Nettogewinne: 5

(1) Nettogewinne = Nettoinvestitionen + Konsum aus Gewinneinkommen

- Ersparnis aus Arbeitseinkommen

+ Außenbeitrag zum Bruttonationaleinkommen.

Auf makroökonomischer Ebene besteht also definitionsgemäß ein positiver $\mathrm{Zu}$ sammenhang zwischen Investitionssumme und Profitsumme. Eine Auseinanderentwicklung beider Größen kann nur durch eine Kompensation durch das Haushaltsdefizit, den Außenbeitrag und/oder den privaten Konsum erfolgen. Hingegen bietet allein der Hinweis auf die positive Aktienkursentwicklung oder die Verschiebung von Real- zu Finanzinvestitionen entgegen populären Ansichten noch keine zufriedenstellende Erklärung dieses Phänomens.

Schließlich lässt sich aus Gleichung (1) eine weitere makroökonomische Restriktion gewinnen, die für die folgenden Betrachtungen wichtig sein wird:

(2) Privater Finanzierungssaldo + Staatlicher Finanzierungssaldo + Finanzierungssaldo des Auslands $=0$. + staatliches Budgetdefizit
Der private Finanzierungssaldo ergibt sich hierbei als Differenz zwischen der Ersparnis aus Gewinnen und Löhnen und den Nettoinvestitionen, der Finanzierungssaldo des Staates als Differenz zwischen Steuereinnahmen und Staatsausgaben und der Finanzierungssaldo des Auslands als Differenz zwischen den Importen des Inlandes und seinen Exporten ins Ausland zuzüglich des Saldos der Faktoreinkommensströme zwischen In- und Ausland. Bildet der private Sektor also vermehrt Überschüsse, indem z. B. seine Investitionen gegenüber der Ersparnis zurückgehen, so müssen die Defizite des Staates und/oder des Auslandes entsprechend ansteigen.

\section{Finanzsystem und wirt- schaftliche Entwicklung in den USA}

Ähnlich wie in anderen Industrieländern setzte in den USA in den 1970er Jahren eine Wachstumsverlangsamung mit steigender Arbeitslosigkeit ein, die die „Golden Age"-Phase der 1950er und 1960er Jahre ablöste. Mitte der 1990er Jahre haben die USA allerdings wieder eine Wachstumsbeschleunigung mit fallenden Arbeitslosenquoten erlebt. Das Wachstum wurde dabei seit den frühen 1980er Jahren in der Tendenz zunehmend vom privaten Konsum getragen, während der Wachstumsbeitrag der privaten Investitionen mit Ausnahme des „New Economy“-Booms tendenziell gesunken ist. Gleichzeitig zeigen die Finanzierungssalden der Sektoren in Abbildung 2 seither größere makroökonomische Ungleichgewichte, die sich in hohen öffentlichen Haushalts- und in Leistungs-

\footnotetext{
Diese ergibt sich aus der Verwendungsrechnung des Bruttoinlandsprodukts (BIP) und der Verteilungsrechnung des Bruttonationaleinkommens (BNE). Das BIP ist definiert als Summe aus Bruttoinvestitionen, Konsum aus Lohneinkommen, Konsum aus Gewinneinkommen, Staatsausgaben und dem Saldo aus Exporten und Importen. Unter Hinzufügung des Saldos der gezahlten und empfangenen Faktoreinkommen an bzw. aus dem Ausland ergibt sich das BNE. Dieses ist im Rahmen der Verteilungsrechnung definiert als Summe aus Nettolöhnen zzgl. Lohnsteuern, Nettogewinnen zzgl. Gewinnsteuern, Abschreibungen sowie dem Saldo aus indirekten Steuern und Subventionen. Aus diesen Zusammenhängen folgt dann unmittelbar Gleichung (1).
} 
bilanzdefiziten sowie seit Mitte der 1990er Jahre in einem mitunter deutlich negativen privaten Finanzierungssaldo niederschlagen.

Die Investitionsneigung der Unternehmen scheint durch Shareholder ValueOrientierung in der jüngeren Vergangenheit in der Tat tendenziell negativ beeinflusst worden zu sein. Neben dem generell $\mathrm{zu}$ erwartenden Einfluss auf die Managementpräferenzen ergab sich über die Finanzierungsseite ein unmittelbar negativer Effekt durch den drastischen Anstieg der Dividendenausschüttungen. ${ }^{6}$ Zeitweise machten diese sogar über $50 \%$ des Nettobetriebsüberschusses bzw. über $100 \%$ der Nachsteuergewinne der nichtfinanziellen Kapitalgesellschaften aus. Zu Beginn der 1980er Jahre stieg auch die Zinsbelastung der Unternehmen an, seit den frühen 1990er Jahren ist diese jedoch wieder rückläufig. Dabei ist das Verhältnis von empfangenen zu geleisteten Zinsen der nichtfinanziellen Kapitalgesellschaften seit Mitte der 1970er Jahre durchweg gestiegen, was auch auf ein - im Vergleich zur realen Investitionstätigkeit - zunehmend lukratives Betätigungsfeld in der Kreditvergabe hindeutet.

Bei der Finanzierung der Bruttoinvestitionen der nichtfinanziellen Kapitalgesellschaften dominieren die internen Finanzierungsmittel (Abbildung 3). Zugleich bestätigen unsere Berechnungen das Ergebnis von Corbett/Jenkinson (1997), nach dem gerade in Ländern mit sogenannten "marktbasierten“ Finanzsystemen (USA, Großbritannien) der Aktienmarkt entgegen populären Vermutungen einen sehr geringen bzw. seit einiger Zeit sogar zunehmend negativen Beitrag zur Investitionsfinanzierung der Unternehmen leistet. Hierin kommt u.a. eine besonders von institutionellen Anlegern mitverantwortete wachsende Bedeutung von Aktienrückkäufen zum Ausdruck (Patgiri et al. 2005). Aktienrückkäufe erhöhen zwar ebenso wie Dividendenausschüttungen ceteris paribus die kurzfristige Aktienrendite eines Unternehmens, sie vermindern aber zugleich die zur Finanzierung der realen Investitionen zur Verfügung stehenden Mittel. Eine weitere Nebenwirkung des negativen Finanzierungsbeitrags von Aktien besteht in der zunehmenden Verschuldung der Unternehmen, die ihre Aktien(rück)käufe zum Teil kreditfinanzieren. Hierin liegt eine Quelle von „finanzieller Fragilität“, da plötzliche Umsatzrückgänge für die Unter-

\section{Abb. 3: Anteil verschiedener Finanzierungsmittel an der Finan- zierung der Bruttoinvestitionen nichtfinanzieller Kapitalge- sellschaften, USA, seit 1960 - in \% -}

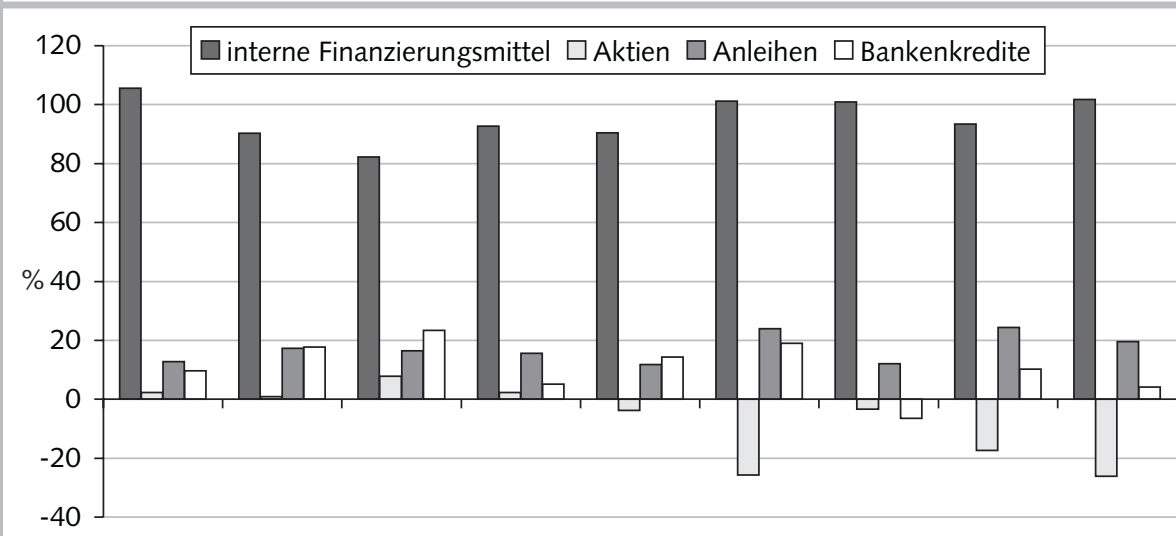

1960-64 1965-69 1970-74 1975-79 1980-84 1985-89 1990-94 1995-99 2000-06

Anmerkungen: Der Beitrag weiterer Finanzierungsmittel und die statistische Diskrepanz bleiben in der Abbildung unberücksichtigt.

Die vollständigen Ergebnisse und methodische Erläuterungen finden sich in van Treeck/Hein/Dünhaupt (2007).

Quelle: Flow of Funds; Berechnungen und Darstellung der Autoren.

WSI MitTEILUNGeN

nehmen mit Insolvenzkrisen verbunden sein können.

Der abnehmenden Investitionsdynamik stand seit Mitte der 1980er Jahre eine im Trend deutlich rückläufige Sparquote der privaten Haushalte gegenüber (von 10-11 \% Anfang der 1980er Jahre auf -1 \% 2006). Wie Tabelle 1 zeigt, ist diese Entwicklung in den 1990er Jahren offenbar auf eine erheblich gestiegene Konsumneigung der reichsten Haushalte zurückzuführen, die von der Einkommensumverteilung und dem Anstieg von Dividenden, Aktienrückkäufen und Vermögenspreisen im Zuge der "Finanzialisierung" besonders profitiert haben (Maki/Palumbo 2001).

Steigende Finanz- und Immobilienvermögen, welche überwiegend auf Bewertungseffekte zurückgehen, ermöglichen die Besicherung von Konsumentenkrediten und Hypotheken, sodass hierüber ein positiver Vermögenseffekt auf den Konsum wirken kann. Ökonometrische Untersuchungen beziffern die marginale Konsumneigung aus Vermögen für die USA auf 4-7 \% (Boone et al. 1998; Maki/Palumbo 2001; Dreger/Slacalek 2007). Allerdings ist durch die zunehmende private Verschuldung die Gefahr gewachsen, dass eine Vermögenspreisdeflation zu erheblichen Liquiditäts- und Solvenzproblemen im privaten Haushaltssektor und bei den Finanzdienstleistern führt. Dies gilt, wie sich jüngst bestätigte, umso mehr für den Immobilienpreisboom der letzten Jahre, als dieser - anders als der Aktienboom der

\begin{tabular}{|c|c|c|}
\hline \multicolumn{3}{|c|}{$\begin{array}{l}\text { Tabelle 1: Sparquote der } \\
\text { privaten Haushalte nach } \\
\text { Einkommensquintilen, USA, } \\
\text { 1992-2000 - in \% - }\end{array}$} \\
\hline \multirow[t]{2}{*}{ Einkommensgruppe } & \multicolumn{2}{|c|}{ Sparquote } \\
\hline & 1992 & 2000 \\
\hline Insgesamt & 5,9 & 1,3 \\
\hline Reichstes Quintil & 8,5 & $-2,1$ \\
\hline 4. Quintil & 4,7 & 2,6 \\
\hline 3. Quintil & 2,7 & 2,9 \\
\hline 2. Quintil & 4,2 & 7,4 \\
\hline Ärmstes Quintil & 3,8 & 7,1 \\
\hline
\end{tabular}

"New Economy“ - in besonderem Maße von einkommensschwachen Haushalten zur Ausweitung von kreditfinanziertem Konsum genutzt wurde (Joint Center for Housing Studies 2006).

Neben dem privaten Konsum wurden die gesamtwirtschaftliche Nachfrage und damit die Profite phasenweise durch hohe staatliche Budgetdefizite von bis zu $6 \%$ des BIP gestützt (Abbildung 2). Während des „New Economy“-Booms Ende der 1990er Jahre konnten zwar kurzzeitig Überschüsse erreicht werden, nach dem Einbruch im Jahr 2000 wurde die gesamtwirtschaftliche Entwicklung aber durch erneute massive Budgetdefizite stabilisiert - anders als bei-

\footnotetext{
6 Die Kausalität kann jedoch wechselseitig sein, und steigende Gewinnausschüttungen können auch Ergebnis fehlender realer Investitionsmöglichkeiten sein (EZB 2007).
} 
Tabelle 2: Leistungsbilanzen ausgewählter Länder - in Mrd. US-Dollar -

\begin{tabular}{lrrr}
\hline & 1996 & $\mathbf{2 0 0 0}$ & $\mathbf{2 0 0 6}$ \\
\hline Industrieländer & & & $-877,6$ \\
USA & $-124,8$ & $-415,2$ & $-55,6$ \\
Vereinigtes Königreich & $-10,5$ & $-37,4$ & 8,1 \\
Euro-Zone & 73,2 & $-36,9$ & $-107,0$ \\
$\quad$ Spanien & $-1,4$ & $-23,0$ & $-56,3$ \\
$\quad$ Italien & 39,5 & $-5,9$ & $-37,1$ \\
$\quad$ Frankreich & 20,8 & 18,2 & 116,8 \\
$\quad$ Deutschland & $-13,8$ & $-33,9$ & 164,9 \\
Japan & 65,1 & 118,7 & $-643,5$ \\
Summe & $-10,7$ & $-404,7$ & \\
\hline Andere & & & 211,3 \\
China (ohne Hongkong) & 7,2 & 20,5 & 2,2 \\
Südkorea & $-23,1$ & 12,3 & 116,8 \\
"Dynamisches Asien“* & $-8,1$ & 61,2 & 45,3 \\
Zentral- und Südamerika & $-36,1$ & $-28,3$ & 280,0 \\
Mittlerer Osten/Afrika & 1,3 & 79,3 & 69,6 \\
Mittel- und Osteuropa & $-0,3$ & 42,4 & 725,3 \\
Summe & $-59,1$ & 187,4 & W SI MITTEILUNGEN \\
\hline *Taiwan, Hongkong, Indonesien, Malaysia, Philippinen, Singapur, Thailand. & & & \\
Quelle: OECD Economic Outlook 80. & & &
\end{tabular}

spielsweise in Deutschland (Hein/Truger 2007).

Vom US-amerikanischen Leistungsbilanzdefizit, das sich in den letzten Jahren auf über $6 \%$ des BIP belief, gehen dämpfende Wirkungen auf Einkommen und Profite aus. Die massiven Kapitalimporte haben jedoch zugleich den „New Economy"-Boom und den auch danach anhaltenden privaten Konsumboom bei gleichzeitigem staatlichen Budgetdefizit finanzierungsseitig ermöglicht. Wie Bernanke (2005) feststellt, weisen in jüngerer Zeit offenbar besonders diejenigen Länder Leistungsbilanzdefizite auf, in denen zugleich starke Vermögenspreissteigerungen stattgefunden haben und die privaten Sparquoten drastisch gesunken sind (Tabelle 2). $\mathrm{Zu}$ den Überschussländern zählen entwickelte Industriestaaten mit schleppender binnenwirtschaftlicher Entwicklung und schwacher Vermögenspreisentwicklung, wie Deutschland oder Japan, aber auch viele Schwellenländer nach Überwindung der Finanzkrisen der 1990er Jahre.

Das US-amerikanische Leistungsbilanzdefizit ist jedoch auf Dauer nicht aufrecht zu erhalten (Godley et al. 2005), vor allem wegen der überwiegend konsumtiven Verwendung der damit verbundenen Kapi-

Abb. 4: Finanzierungssalden als Anteil am nominalen Bruttoinlandsprodukt, Deutschland, seit 1960 - in \% -

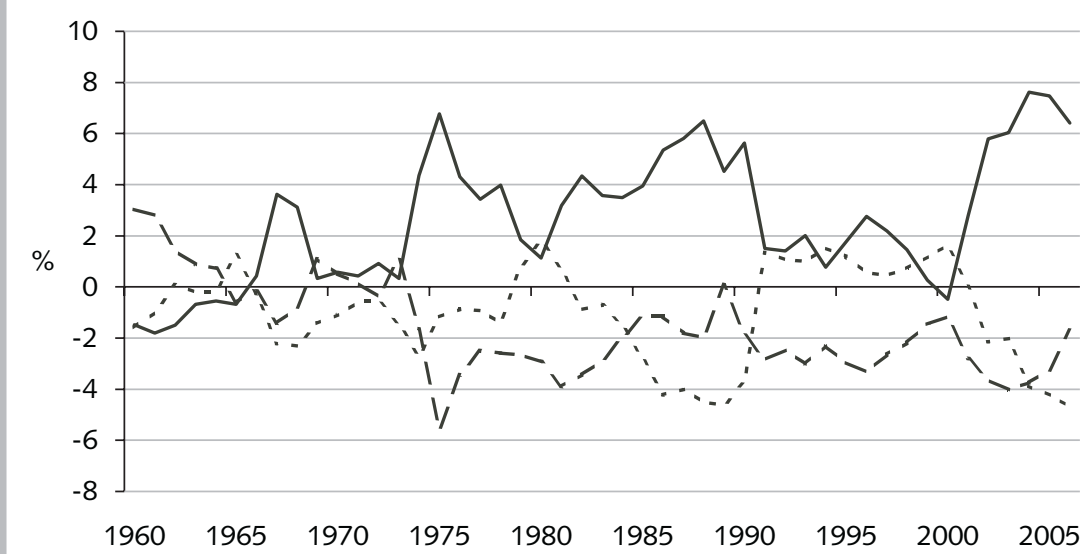

$$
\begin{array}{|l}
\hline \text { - privater Finanzierungssaldo } \\
-\ldots \text { staatlicher Finanzierungssaldo } \\
\ldots-\ldots \text { - Finanzierungssaldo des Auslands }
\end{array}
$$

talimporte. Dies könnte zulasten zukünftiger Wachstumsmöglichkeiten gehen und damit zulasten der glaubhaften Fähigkeit, die mit den Kapitalimporten verbundenen Zahlungsverpflichtungen zu bedienen.

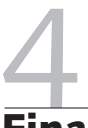

\section{Finanzsystem und wirt- schaftliche Entwicklung in Deutschland}

Wie in anderen entwickelten Industrieländern schwächte sich das BIP-Wachstum auch in Deutschland nach der „GoldenAge"-Phase der 1950er und 1960er bereits in den 1970er Jahren und dann insbesondere in den 1980er Jahren deutlich ab. Anfang der 1990er Jahre erlebte Deutschland dann im Rahmen des Vereinigungsbooms eine Wachstumsbeschleunigung, die ab Mitte der 1990er Jahre in eine stagnative Phase mündete. Angesichts der institutionellen Entwicklung des Finanzsystems ist nur für diese letzte Phase ein Einfluss der Finanzialisierungsprozesse denkbar. „Finanzialisierung " und Shareholder ValueOrientierung haben erst mit der Deregulierung des Finanzsystems und der politisch vorangetriebenen Auflösung der „Deutschland $\mathrm{AG}^{\text {“ }}$ seit Mitte/Ende der 1990er Jahre an Bedeutung gewonnen (Windolf 2005). So können beispielsweise Unternehmen seit 1998 Aktienrückkäufe tätigen, nachdem dies seit der Finanzkrise von 1931 nicht mehr gestattet gewesen war. Im Januar 2002 wurde zudem die Steuer auf Gewinne aus Beteiligungsveräußerungen bei Kapitalgesellschaften abgeschafft.

Im Gegensatz zu den USA weist Deutschland während der 1980er Jahre systematisch einen (steigenden) Leistungsbilanzüberschuss auf (Abbildung 4). In der Folge der Wiedervereinigung verschlechterte sich die Außenhandelsposition zwar zeitweise, seit 2000 setzt sich der positive Trend der 1980er Jahre aber fort, und der Leistungsbilanzüberschuss erreichte zuletzt über $4 \%$ des BIP. In deutlichem Unterschied zu den USA lassen sich in Deutschland seit Mitte der 1970er Jahre zudem dauerhafte Finanzierungsüberschüsse des privaten Sektors feststellen. Nach einer vorübergehenden Verschlechterung während der 1990er Jahre im Zuge des Wiedervereinigungs- und des (im Vergleich zu den USA verhalten ausfallenden) „New Economy“-Booms hat der private Finanzie- 
rungssaldo in der jüngsten Vergangenheit einen Überschuss von bis zu knapp 8 \% des BIP erreicht. Hierin spiegelt sich die seit vielen Jahren schwache binnenwirtschaftliche Konsum- und Investitionsnachfrage wider.

Die seit den frühen 1980er Jahren - mit kurzer Unterbrechung während der außergewöhnlichen 1990er Jahre - anhaltende Verbesserung der Wettbewerbsfähigkeit der deutschen Exportproduktion geht wesentlich auf die im internationalen Vergleich sehr moderate Lohnstückkostenentwicklung zurück (Hein/Truger 2007), die mit einer drastischen Einkommensumverteilung zulasten der Arbeitseinkommen verbunden ist. Diese geht allerdings, anders als in den USA, bisher nicht mit einer deutlichen Verschiebung der Profite zugunsten der finanziellen und zulasten der nichtfinanziellen Kapitalgesellschaften einher.

Mit Blick auf das Investitionsverhalten der nichtfinanziellen Kapitalgesellschaften lässt sich feststellen, dass der Anteil der Ausschüttungen und Entnahmen am Betriebsüberschuss seit den 1980er Jahren bis in die jüngste Vergangenheit nahezu konstant geblieben ist. Hierüber scheint also zumindest bei aggregierter Betrachtung kein negativer Einfluss von Shareholder Value-Orientierung auf die Investitionstätigkeit erkennbar. Der Anteil der Nettozinszahlungen weist, anders als in den USA, bereits seit Anfang der 1980er Jahre einen sinkenden Trend auf. Zudem lässt sich ähnlich wie in den USA über den Zeitraum 1980-2006 ein Anstieg des Verhältnisses von empfangenen zu geleisteten Zinsen der nichtfinanziellen Kapitalgesellschaften feststellen.

Interne Finanzierungsmittel spielen auch im „bankenbasierten“ Finanzsystem Deutschlands eine herausragende Rolle für die Finanzierung der Bruttoinvestitionen der nichtfinanziellen Kapitalgesellschaften (Abbildung 5; siehe hierzu auch Corbett/ Jenkinson 1997). Erst dann folgen mit großem Abstand Bankkredite, die jedoch einen größeren Beitrag leisten als in den USA. In den letzten Jahren haben handelbare Unternehmensanleihen an Bedeutung gewonnen. Der Nettofinanzierungsbeitrag des Aktienmarktes war in Deutschland traditionell unbedeutend. Ab Mitte der 1990er Jahre wurde er jedoch erstmals über einen längeren Zeitraum negativ, unter anderem, weil viele Unternehmen von der gesetzlichen Neuregelung Gebrauch machten, eigene Aktien zurückkaufen zu dürfen (EZB

\section{Abb. 5: Anteil verschiedener Finanzierungsmittel an der Finan- zierung der Bruttoinvestitionen nichtfinanzieller Kapitalgesell- schaften, Deutschland, seit 1960 - in \% -}

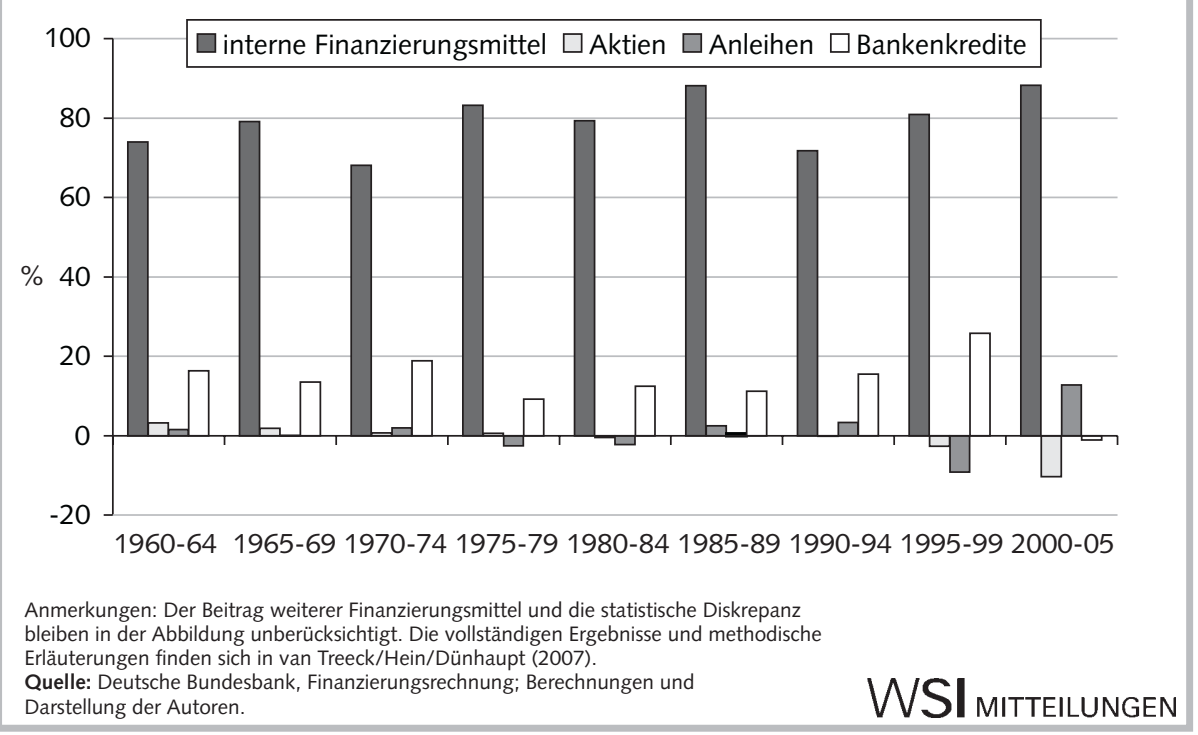

2007). Gleichzeitig ist die Innenfinanzierungsquote der Bruttoinvestitionen auf fast 90 \% gestiegen, während die Unternehmen Kredite getilgt bzw. an andere Sektoren vergeben haben. Diese noch jungen Entwicklungen lassen zumindest Zweifel daran entstehen, dass die Liberalisierung des Finanzsystems in Deutschland einen positiven Volumeneffekt für die Investitionstätigkeit mit sich bringt (EZB 2007).

Anders als in den USA ist die schwache Investitionsdynamik in Deutschland nicht durch den privaten Konsum kompensiert worden. Nachdem die Sparquote der privaten Haushalte seit Mitte der 1960er Jahre lange Zeit auf einem Niveau von ca. 12-14\% verharrte, fiel sie Ende der 1990er Jahre kurzzeitig auf unter $10 \%$, stieg aber seit 2000 wieder an. Insbesondere die Sparquote der einkommensstärksten und vermögenden Haushalte weist ein deutlich höheres Niveau auf als in den USA und lässt trotz Anstiegs der privaten Vermögen auch keine Tendenz nach unten erkennen. In den unteren Einkommensgruppen ist die Ersparnisbildung hingegen auch in Deutschland regelmäßig negativ (Tabelle 3).

Ökonometrische Untersuchungen kommen für Deutschland in der Regel zu weniger signifikanten und niedrigeren Werten für die Konsumneigung aus Aktien- und Immobilienvermögen als für die USA (Boone et al. 1998; Dreger/Slacalek 2007). Zudem haben sich hierzulande - ganz anders als in den USA und anderen Industrieländern - die Immobilienpreise überaus schwach entwickelt. Die Entwicklung der Sparquote wird daher durch die Umverteilung zulasten der Arbeitseinkommen und durch die mit der Politik der ,strukturellen Reformen" (Agenda 2010, Hartz-Gesetze, Erhöhung des Renteneintrittsalters) verbundene Verunsicherung der privaten Haushalte dominiert (Klär/Slacazek 2006).

Die schwache Entwicklung der Binnennachfrage und die hohen Finanzierungsüberschüsse des privaten Sektors, insbesondere seit 2000/01, schlagen sich in einem dauerhaften staatlichen Defizit sowie in noch stärkerem Umfang in Leistungsbilanzüberschüssen und damit in Kapitalexporten an das Ausland nieder (Tabelle 2). Die negativen Finanzierungssalden des Staates sind im Falle Deutschlands dabei nicht Ausdruck einer aktiv stabilisierenden Nachfragepolitik, sondern ergeben sich makroökonomisch aus den Versuchen ei-

\begin{tabular}{|c|c|}
\hline \multicolumn{2}{|c|}{$\begin{array}{l}\text { Tabelle 3: Sparquote der } \\
\text { privaten Haushalte und } \\
\text { Einkommensquintile, } \\
\text { Deutschland } 2003 \text { - in } \% \text { - }\end{array}$} \\
\hline Einkommensgruppe & Sparquote \\
\hline Insgesamt & 11,6 \\
\hline Reichstes Quintil & 19,0 \\
\hline 4. Quintil & 11,0 \\
\hline 3. Quintil & 6,0 \\
\hline 2. Quintil & 1,9 \\
\hline Ärmstes Quintil & $-4,6$ \\
\hline $\begin{array}{l}\text { Quelle: Klär/Slacalek } \\
(2006) \text { auf Crundlage } \\
\text { der EVS } 2003 .\end{array}$ & TTEILUNGEN \\
\hline
\end{tabular}


ner Haushaltskonsolidierung durch Ausgabenbeschränkungen in einer ökonomischen Schwächephase (Hein/Truger 2007).

Hohe Leistungsbilanzüberschüsse und der damit verbundene Kapitalexport der letzten Jahre implizieren, dass von Deutschland aus vermehrt Finanzinvestitionen im Ausland getätigt wurden. Vor diesem Hintergrund kann auch die massive Beteiligung deutscher Banken an der Finanzierung US-amerikanischer Hypothekenkredite während der vergangenen Jahre verstanden werden: Während in Deutschland die Anlagemöglichkeiten angesichts der binnenwirtschaftlichen Wachstumsschwäche begrenzt waren, bot sich in Ländern mit kräftigerer Binnennachfrage und noch stärker deregulierten Finanzmärkten, wie den USA, eine Vielzahl scheinbar lukrativer Anlageoptionen. Hieraus folgt dann aber auch eine verstärkte Gefährdung der Finanzmarktentwicklung in Deutschland durch Finanzkrisen im Ausland.

\section{Chancen und Risiken der "Finanzialisierung"}

In den USA ist „Finanzialisierung“ während der letzten zweieinhalb Jahrzehnte mit einer tendenziellen Abschwächung der Investitionstätigkeit (mit Ausnahme des außergewöhnlichen „New Economy“Booms) einhergegangen. Gleichzeitig haben die Profiteure von Dividendenausschüttungen, Aktienrückkäufen und steigenden Finanzvermögen ihre Sparquote drastisch vermindert. Im Ergebnis zeigten sich eine - trotz schwacher Investitions- tätigkeit - positive Entwicklung der Unternehmensgewinne und eine bisher relativ robuste gesamtwirtschaftliche Dynamik.

Allerdings kann bezweifelt werden, dass die sehr stark vom privaten Konsum getragene makroökonomische Entwicklung in den USA als Vorbild für Deutschland herangezogen werden kann oder sollte. Zum einen ist der Konsumboom der jüngeren Vergangenheit weitgehend auf das veränderte Sparverhalten der reichsten Haushalte zurückzuführen, während sich die Einkommensungleichheit massiv verstärkt hat. Der Immobilienpreisboom der letzten Jahre ermöglichte zwar verstärkt auch einkommensschwachen Haushalten zunehmende Konsummöglichkeiten trotz stagnierender Einkommen, allerdings häufig um den Preis der latenten Überschuldung. Zum anderen sind der andauernde Absturz der privaten Sparquote, die hohe Verschuldung der privaten Haushalte sowie die hohe Auslandsverschuldung mit einer erheblichen Fragilität des nationalen und internationalen Finanzsystems verbunden.

In Deutschland sind die Wirkungen der „Finanzialisierung" bislang weitaus weniger deutlich. Auch hier ist es nach dem Wiedervereinigungsboom zu einer tendenziellen Abschwächung der privaten Investitionstätigkeit gekommen - zusammen mit ersten Finanzialisierungstendenzen im Unternehmenssektor. Allerdings stand die unverändert hohe Sparquote der einkommensstarken und vermögenden Haushalte - zusammen mit einem deutlich restriktiveren makroökonomischen Politik-Mix und einer deutlicheren Umverteilung zulasten der Arbeitseinkommen - bisher einem nachhaltigen Anstieg der Konsum- nachfrage im Wege. Eine Auseinanderentwicklung von Profiten und Investitionen in Deutschland wird wesentlich durch öffentliche Haushaltsdefizite und durch Leistungsbilanzüberschüsse ermöglicht, bei allerdings deutlich geringerem Wachstum als in den USA.

Die aktuellen, von der Krise am USamerikanischen Immobilienmarkt ausgelösten Turbulenzen auf den internationalen Finanzmärkten verdeutlichen die Gefahren eines finanzmarktzentrierten Wachstumsmodells. Obwohl sich ein solches Modell in Deutschland bisher nicht voll entwickelt hat, ist auch die wirtschaftliche Entwicklung hierzulande von diesen Gefahren betroffen. Die Fragilität der exportgetriebenen wirtschaftlichen Entwicklung in Deutschland ergibt sich dabei bei einem möglichen wirtschaftlichen Einbruch in den USA zum einen direkt über den Exportkanal, zum anderen aber auch über Ansteckungen durch die internationalen Finanzmärkte.

Wirtschaftspolitisch folgt hieraus, eine stärkere binnenwirtschaftlich ausgerichtete Entwicklung in Deutschland zu forcieren und eine stärkere Ausrichtung des Finanzsektors auf die langfristige Finanzierung privater Investitionen im Inland anzustreben. Eine solche wirtschaftspolitische Strategie sollte deshalb eine Neuausrichtung der makroökonomischen Politik, d.h. der Geld-, Fiskal- und Lohnpolitik, ${ }^{7}$ mit einer Re-Regulierung des Finanzsektors ${ }^{8}$ verbinden.

\footnotetext{
Vgl. hierzu ausführlicher z. B. Hein/Truger (2007) und Hein et al. (2005).

8 Vgl. hierzu z. B. die Vorschläge in Palley (2007).
} 
Akerlof, G. (2007): The missing motivation in macroeconomics, in: American Economic Review 1, S. 5-36

Bernanke, B. (2005): The Global Saving Glut and the U. S. Current Account Deficit, Remarks at the Homer Jones Lecture, 14.04.

Boone, L./Giorno, C./Richardson, P. (1998): Stock Market Fluctuations and Consumption Behaviour: Some Recent Evidence, OECD Working Paper 208

Corbett, J./Jenkinson, T. (1997): How is investment financed? A study of Germany, Japan, the United Kingdom and the United States, in: Manchester School, Supplement, S. 69-93

Dreger, C./Slacalek, J. (2007): Finanzmarktentwicklung, Immobilienpreise und Konsum, DIW-Wochenbericht 35

Europäische Zentralbank (EZB) (2007): Aktienrückkäufe im Euroraum, in: Monatsbericht Juni, S. 111-120

Godley, W./Papadimitriou, D./Dos Santos, C./Zezza, G. (2005): The United States and Her Creditors, Can the Symbiosis Last? Levy Economics Institute of Bard College, Strategic Analysis

Graham, J./Harvey, C./Rajgopal, S. (2005): The economic implications of corporate financial reporting, in: Journal of Accounting and Economics 1-3, S. 3-73

Hein, E. (2004): Verteilung und Wachstum. Eine paradigmenorientierte Einführung unter besonderer Berücksichtigung der post-keynesianischen Theorie, Marburg

Hein, E. (2005): Finanzstruktur und Wirtschaftswachstum - theoretische und empirische Aspekte, IMK Studies 1

Hein, E./Horn, G./Tober, S./Truger, A. (2005): Eine gesamtwirtschaftliche Politik-Strategie für mehr Wachstum und Beschäftigung, in: WSI-Mitteilungen 8, S. 411-418

Hein, E./Truger, A. (2007): Die deutsche Wachstums- und Beschäftigungsschwäche im europäischen Kontext - ein Lehrstück makroökonomischen Missmanagements, in: Chaloupek, G./Hein, E./Truger, A. (Hrsg.): Ende der Stagnation? Wirtschaftspolitische Perspektiven für mehr Wachstum und Beschäftigung in Europa, Wien, S. 43-63
Hubbard, R. G. (1998): Capital-market-imperfections and investment, in: Journal of Economic Literature 1, S. 193-225

Jensen, M. C./Meckling, W. H. (1976): Theory of the firm - managerial behavior, agency costs and ownership structure, in: Journal of Financial Economics 4, S. 305-360

Joint Center for Housing Studies (2006): The State of the Nation's Housing, Report of the Joint Center for Housing Studies of Harvard University

Klär, E./Slacalek, J. (2006): Entwicklung der Sparquote in Deutschland: Hindernis für die Erholung der Konsumnachfrage, DIW-Wochenbericht 40

Levine, R. (2005): Finance and growth: theory and evidence, in: Aghion, P./Durlauf, S. (Hrsg.): Handbook of Economic Growth, Amsterdam, S. 865-934

Maki, D. M./Palumbo, M. G. (2001): Disentangling the Wealth Effect: A Cohort Analysis of Household Saving in the 1990s, Board of Governors of the Federal Reserve System, Finance and Economics Discussion Series 21

Palley, T. (2007): Expand Sarbox, Not Shrink It, http://www.thomaspalley. $\mathrm{com} / \mathrm{p}=68$

Patgiri, R./Gaspar, J.-M./Massa, M./Matos, P./Rehman, Z. (2005): Can Buybacks Be a Product of Shorter Shareholder Horizons?, CEPR Discussion Paper 4813

van Treeck, T./Hein, E./Dünhaupt, P. (2007): Finanzsystem und wirtschaftliche Entwicklung: Tendenzen in den USA und in Deutschland aus makroökonomischer Perspektive, IMK Studies 5

Windolf, P. (Hrsg.) (2005): Finanzmarktkapitalismus: Analysen zum Wandel von Produktionsregimen, Wiesbaden 